\title{
Doble Inervación del Músculo Braquial en la Población Chilena
}

\author{
Dual Innervation of the Brachialis Muscle in the Chilean Population
}

\author{
*Claudio Molina; **** Cristián Uribe; "Álvaro Heras; ** Cristián Astorga; \\ "Jorge Lemus \& *Alberto Rodríguez
}

\begin{abstract}
MOLINA, C.; URIBE, C.; HERAS, A.; ASTORGA, C.; LEMUS, J. \& RODRÍGUEZ, A. Doble inervación del músculo braquial en la población chilena. Int. J. Morphol., 29(4):1207-1211, 2011.

RESUMEN: La doble inervación del músculo braquial está dada por el nervio musculocutáneo y por ramos provenientes del nervio radial según lo describen algunos textos anatómicos y numerosos trabajos científicos. Sin embargo, no existe consenso en la frecuencia con la que ésta se presenta. El objetivo de este estudio es verificar la presencia y determinar la frecuencia de la contribución del nervio radial en la inervación del músculo braquial. En el presente trabajo se utilizaron 30 miembros superiores previamente fijados con solución fijadora y conservadora. Se realizó disección de la región braquial para determinar la presencia de ramos del nervio radial inervando al músculo braquial, verificando la presencia de ramos que penetraban en el músculo y tomando muestras de estos ramos en el sitio donde perforaban al músculo para su estudio histológico. Fue posible observar la presencia de ramos del nervio radial que penetraban en el músculo braquial en un $90 \%$ de los casos; en ellos el punto motor de estos ramos se ubicó siempre en el tercio distal del músculo. De acuerdo a los resultados obtenidos en el presente trabajo, la doble inervación del músculo braquial observada por autores clásicos y reportada en estudios en diversas poblaciones, está presente en alto porcentaje de la muestra. Estos hallazgos pueden servir de referencia para procedimientos quirúrgicos, estudios electromiográficos y sonográficos.
\end{abstract}

PALABRAS CLAVE: Músculo Braquial; Nervio Radial; Plexo Braquial.

\section{INTRODUCCIÓN}

El músculo braquial se ubica en el compartimento anterior de la región braquial y constituye el principal músculo flexor de codo. La inervación de este músculo está dada por el nervio musculocutáneo como lo describen algunos textos anatómicos (Feneis \& Dauber, 2000; Moore \& Dalley, 2006; Latarjet \& Ruiz Liard, 2008; Gray \& Standring, 2005). Sin embargo, textos anatómicos antiguos (Cloquet, 1830; Hirschfeld, 1866; Testut, 1884; Le Double, 1897; Poirer et al., 1901; Testut \& Latarjet 1944; Lewis, 1944; Brash \& Jamieson, 1949; Lockhart \& Hamilton, 1959; Williams \& Warwick, 1975) y modernos (Jenkins \& Hollinshead, 2002; Rouvière, 2007) dan cuenta de una doble inervación del músculo braquial caracterizada por la contribución del nervio radial en la inervación de este músculo, pero sin detallar su frecuencia de aparición.

La presencia de ramos del nervio radial que abordan el músculo braquial se haya también descrita en numerosos trabajos científicos (Ip \& Chang, 1968; Yan et al., 1998; Ji \&
Chung, 2002; Mahakkanukrauh \& Somsarp, 2002; Spinner et al., 2003; Pacha Vicente et al., 2005; Blackburn et al., 2007; Park et al., 2007; Frazer et al., 2007; Oh et al., 2009; Prakash et al., 2009). Estos trabajos, sin embargo, difieren en los resultados encontrados, reportando entre un $67 \%$ y un $100 \%$ los casos la doble inervación del músculo braquial.

Las diferencias encontradas en estos trabajos podrían obedecer a la disparidad en las metodologías utilizadas entre ellos, particularmente, el tamaño muestral utilizado el cual varió entre 16 y 152 miembros superiores estudiados.

Las investigaciones que se han centrado en la inervación del músculo braquial, concuerdan respecto al lugar de penetración del ramo muscular proveniente del nervio radial (Ji \& Chung; Mahakkanukrauh \& Somsarp; Oh et al.; Prakash et al.). En estos trabajos se señala que este punto motor se ubicaría, en la mayoría de los casos, en el tercio distal del músculo braquial.

\footnotetext{
* Programa de Anatomía y Biología del Desarrollo, Facultad de Medicina Universidad de Chile, Chile.

** Programa de Magister, Facultad de Medicina Universidad de Chile, Chile.

${ }^{* * * *}$ Facultad de Medicina, Universidad Finis Terrae, Chile.
} 
Los ramos musculares del nervio radial que participan en la inervación del músculo braquial presentan relevancia quirúrgica debido a que, por su ubicación, están expuestos a ser lesionados durante el abordaje anterolateral del húmero (Jordan \& Mirzabeigi, 2000; Browner et al., 2009). El objetivo del presente estudio es verificar la presencia y determinar la frecuencia de la contribución del nervio radial en la inervación del músculo braquial en una muestra realizada de la Facultad de Medicina de la Universidad de Chile.

\section{MATERIAL Y MÉTODO}

En este estudio fueron utilizados 30 miembros superiores provenientes de 15 cadáveres ( 4 femeninos y 11 masculinos) previamente fijados mediante solución fijadora conservadora procedentes del Programa de Anatomía y Biología del Desarrollo, de la Facultad de Medicina de la Universidad de Chile. El rango de edad de los individuos fue entre 20 y 80 años.

Mediante una incisión en la línea mediana de la región braquial se expuso la tela subcutánea de la región braquial anterior y posterior desde la región cubital hasta la región axilar. Se observó el músculo braquial ubicado en el compartimento anterior de la región braquial y se identificaron los ramos del nervio musculocutáneo que se dirigen a este músculo. Se reconoció el septo intermuscular lateral así como el surco bicipital lateral entre los músculos bíceps braquial y braquiorradial.

Se examinó el nervio radial en su recorrido a través del surco del nervio radial en el húmero, entre las cabezas medial y lateral del músculo tríceps braquial, y en el surco entre los músculos braquial y braquiorradial. Posteriormente este nervio se disecó cuidadosamente a lo largo de la región braquial.

Se buscó ramos del nervio radial que se dirigían al músculo braquial, y en los casos presentes, éstos fueron fotografiados con cámara Canon Digital Rebel XTi 10.1MP Digital.

Análisis histológico: Para confirmar la presencia de tejido nervioso se seleccionaron tres muestras al azar de ramos del nervio radial los cuales fueron separados de este mismo nervio. Posteriormente fueron fijados en formol calcio $10 \%$ y embebidos en parafina para ser cortados en secciones transversales de 5 um de diámetro a analizar; luego se procedió a su tinción con hematoxilina eosina para su análisis con microscopio de luz Leica EZ4 HD con cámara digital incorporada.

\section{RESULTADOS}

Origen de los ramos para el músculo braquial. Se observó el origen de los ramos musculares para la inervación del músculo braquial. En 30 (100\%) de los casos el nervio musculocutáneo aportó ramos al músculo braquial ingresando desde proximal hacia distal. En 27 (90\%) de los casos el nervio radial participó en la inervación del músculo braquial ingresando lateralmente hacia la porción distal del músculo braquial (Fig. 1).

Origen de los ramos para el musculo braquial según lateralidad. Del total de los 30 casos analizados, en los miembros superiores del lado derecho el músculo braquial estaba inervado por el nervio musculocutáneo en todos los casos $(100 \%)$ y 14 casos $(93,3 \%)$ recibieron inervación por parte del nervio radial. Las muestras provenientes del lado izquierdo todas $(100 \%)$ recibieron inervación por parte del nervio musculocutáneo y en 13 casos $(86,7 \%)$ estaban inervados por el nervio radial.

Origen de ramos musculares para el músculo braquial según sexo. Se observó que en los 8 miembros superiores provenientes de cadáveres femeninos la inervación del músculo braquial se realizó por los nervios musculocutáneo y radial. Los 22 miembros superiores provenientes de cadáveres masculinos, 10 de ellos correspondientes a los del lado derecho, presentaron doble inervación y 9 miembros superiores izquierdos presentaron doble inervación.

Análisis Histológico. La observación de los cortes histológicos permitió reconocer fascículos musculares del músculo braquial, así como también la presencia de tejido nervioso en el perimisio del músculo. La morfología del tejido nervioso observado fue compatible con la de un nervio, el cual se presentó acompañado de estructuras vasculares (Fig. 2).

\section{DISCUSIÓN}

Nuestras observaciones sugieren que el músculo braquial presenta una doble inervación establecida por el nervio musculocutáneo y el nervio radial en un alto porcentaje $(90 \%)$ de la muestra estudiada. Estos resultados se acercan a estudios realizados por Ip \& Chang en población China el cual reportó que $100 \%$ de casos estudiados presentaron doble inervación del músculo braquial; de la misma forma lo reportó Yan et al. en población japonesa. Otros trabajos como el de Mahakkanukrauh \& Somsarp informan de un 


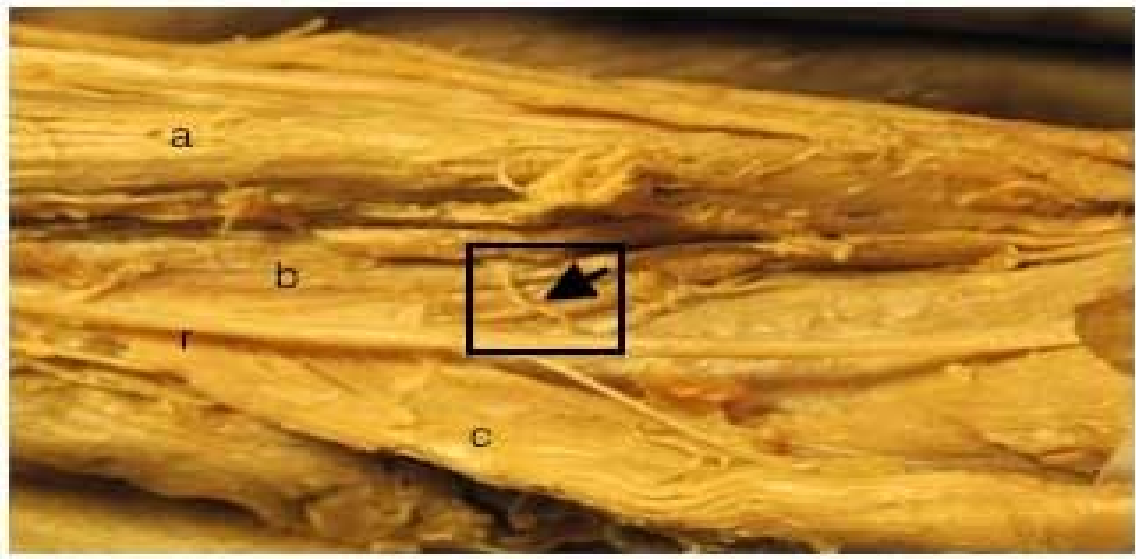

Fig. 1. Miembro Superior derecho de individuo adulto de sexo masculino. Plano profundo de la región braquial. a Músculo Bíceps Braquial. b Músculo Braquial. c Músculo Braquiorradial. r Nervio Radial. Flecha. Ramo muscular del nervio radial. El rectángulo corresponde al ramo que se observa en la Figura 2.

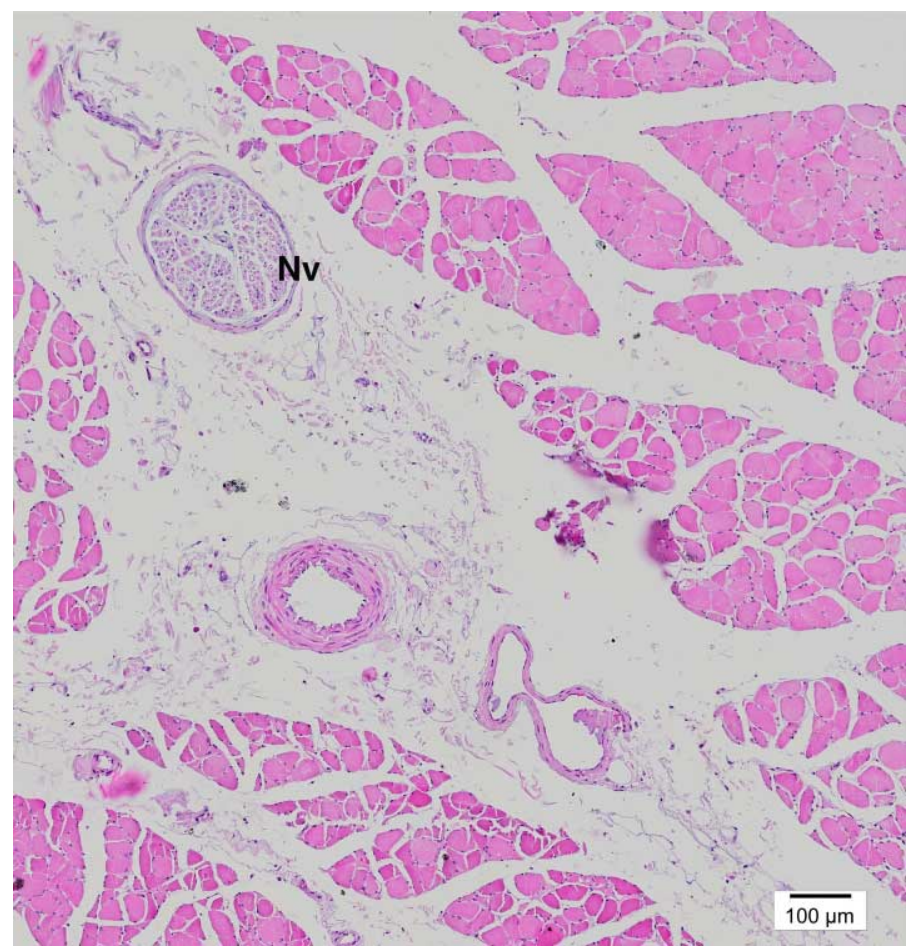

Fig. 2. Corte transversal del ramo muscular del Nervio Radial (Nv) próximo a su entrada al Músculo Braquial. H.E. 20x

$81,6 \%$ en población tailandesa y Prakash et al. 72,14\%, en población de la India.

Sin embargo, autores como Blackburn et al., Frazer et al. informan valores más bajos, en estudios en cadáveres caucásicos en el Reino Unido. Las diferencias en estos estudios podrían obedecer a los diversos tamaños muestrales utilizados.

Por otro lado, existe controversia acerca del origen del ramo del nervio radial. Oh et al. en una reciente publicación acerca del origen del ramo del nervio radial inervando el músculo braquial, determinó que este ramo tiene su origen en la raíz C5 en 5 casos, C6 en 11 casos, C5,C6 en 3 casos y C6, C7 en 1 caso, y observó que el ramo estaba incluido en el tronco superior o medio y sigue por el fascículo posterior del plexo braquial en todos los casos analizados (20). Esta descripción contrasta con Yan et al. que afirmó que este ramo proviene del fascículo anterior y tiene su origen en las raíces C6, C7, en un estudio que analizó 16 casos. Determinar el origen del ramo del nervio radial que inerva al musculo braquial no fue objetivo de este estudio.

Desde la biología del desarrollo la literatura reporta escasas evidencias frente al origen de una doble inervación. Esto ha sido discutido en relación a la inervación del músculo braquial haciendo referencia al origen embriológico de éste. Es así como los autores Mahakkanukrauh \& Somsarp, han sugerido que la porción distal del músculo braquial tendría su origen de la masa premuscular dorsal en el embrión, apoyados en los estudios de Sunderland, (1945) que en secciones transversales del nervio radial observó que el ramo para el músculo braquial se encontraba localizado con el ramo para el músculo braquiorradial, lo que reflejaría una relación directa entre la porción inferior del músculo braquial y el musculo braquiorradial. Sin embargo, no hay estudios que hayan abordado esta interrogante.

A pesar de la existencia de diversos trabajos en variadas poblaciones, desde el punto de vista clínico, no se ha abordado la importancia de esta inervación. Jones (1919) describió contracción palpable y localizada, en una lesión completa del nervio musculo-cutáneo en la cual el nervio radial permanecía intacto. Spinner et al. mediante resonancia magnética observó una atrofia de la porción distal del músculo braquial en dos pacientes con parálisis del nervio radial.

En este estudio hemos evidenciado que la doble inervación del musculo braquial está presente en un alto porcentaje de la población chilena y enfatizamos la necesidad de otorgar importancia a esta condición ya que es un antecedente importante a considerar en abordajes quirúrgicos y en la rehabilitación de pacientes que presenten lesiones nerviosas del miembro superior. 
AGRADECIMIENTOS: Nuestros agradecimientos a todas las personas que hicieron posible la realización de esta investigación principalmente al Dr. Ignacio Roa de la Universidad de Talca por su apoyo en la edición, al Dr. Octavio Binvignat de la Universidad Autónoma por sus aportes en las referencias bibliográficas, al Prof.
Miguel Soto, William Aguilar, de la Facultad de Medicina de la Universidad de Chile por su ayuda con los preparados histológicos y a las alumnas ayudantes Josefina Loeser, Trinidad Pascual y María José Forteza por su apoyo en los procedimientos de toma de muestras.

MOLINA, C.; URIBE, C.; HERAS, A.; ASTORGA, C.; LEMUS, J. \& RODRÍGUEZ, A. Dual innervation of the brachialis muscle in the Chilean population. Int. J. Morphol., 29(4):1207-1211, 2011.

SUMMARY: The dual innervation of the brachialis muscle by the musculocutaneos nerve and branches from the radial nerve has been described by some anatomical texts and several scientific papers; however, there is no consensus about the frequency with which this occurs. In this study we proposed to check the presence and determine the frequency of the contribution of radial nerve in the innervations of the brachialis muscle. In this study we used 30 upper limbs fixed in fixative and conservative solution. Dissection of the brachial region was carried out, checking the branches that penetrated the muscle and taking samples of these branches to histological study. It was possible to observe the presence of branches of the radial nerve innervating the brachialis muscle in a $90 \%$ of the sample used in this study; the motor point of these branches was always located in the distal third of this muscle. According with the results of this study, the dual innervations of the brachial muscles observed by classical authors and reported by several investigations is also present in a high percentage of the sample. These findings can serve as reference for surgical procedures, electromyographic and ultrasound studies.

KEY WORDS: Brachialis muscle; Radial nerve; Brachial plexus.

\section{REFERENCIAS BIBLIOGRAFICAS}

Blackburn, S. C.; Wood, C. P.; Evans, D. J.; \& Watt, D. J. Radial nerve contribution to brachialis in the UK Caucasian population: Position is predictable based on surface landmarks. Clin. Anat., 20:64-7, 2007.

Brash, M. A. \& Jamieson, E. B. (eds.). Anatomía humana de Cunningham. Barcelona, Manuel Marín, 1949.

Browner, B. D.; Jupiter, J. B.; Levine, A. M. \& Trafton, P. G. Skeleta Trauma: Basic Science, Management and Reconstruction. 4 ed. Philadelphia, Elsevier Science, 2009. V. 1.

Cloquet, H. A system of human anatomy. Boston, Wells and Lilly, 1830.

Frazer, E. A.; Hobson, M. \& McDonald, S. W. The distribution of the radial and musculocutaneous nerves in the brachialis muscle. Clin. Anat., 20:785-9, 2007.

Feneis, H. \& Dauber, W. Pocket Atlas of Human Anatomy. Based on the International Nomenclature. $14^{\circ} \mathrm{ed}$. Stuttgart, Thieme, 2000.

Gray, H. \& Standring, S. Gray's anatomy: The anatomical basis of clinical practice. $39^{\circ}$ ed. Edinburgh, UK, Elsevier Churchill Livingstone, 2005.

Hirschfeld, L. Nèvrologie et esthésiologie. Traité et iconographie du système nerveux et des organes des sens de l'homme avec leur mode de preparation. $2^{\circ}$ ed. Paris, Masson, 1866.

Ip, M. C. \& Chang, K. S. A study on the radial supply of the human brachialis muscle. Anat. Rec., 162:363-71, 1968.
Jenkins, D. B. \& Hollinshead, W. H. Hollinshead's functional anatomy of the limbs and back. $8^{\circ}$ ed. Saunders, 2002

Ji, H. J. \& Chung, I. H. The muscular branch of the radial nerve to the brachialis muscle in Korean. Korean J. Phys. Anthropol., 15:127-31, 2002.

Jones, F. W. Voluntary muscle movements in cases of nerve lesions. J. Anat., 54:41-57, 1919.

Jordan, C. \& Mirzabeigi, E. Atlas of Orthopaedic Surgical Exposures. New York, Thieme, 2000.

Mahakkanukrauh, P. \& Somsarp, V. Dual innervation of the brachialis muscle. Clin. Anat., 15:206-9, 2002.

Moore, K. L. \& Dalley, A. F. Clinically oriented anatomy. 5th ed. Philadelphia, Lippincott Williams \& Wilkins, 2006.

Latarjet, M. \& Ruiz Liard, A. Anatomía Humana. $4^{\text {ta }}$ ed. Buenos Aires. Ed. Panamericana, 2004.

Le Double, A. F. Traité des variations système musculaire de l' home et de leur signification au point de vue de l'anthropologie zoologique. Paris, Schleicher frères, 1897.

Lewis W. H. Gray's Anatomy of the human body. $24^{\circ}$ ed. Philadelphia, Lea \& Febiger, 1944.

Lockhart, R. D.; Hamilton, G. F. \& Fyfe, F. W. Anatomy of the Human Body. $2^{\circ}$ ed. Philadelphia, Lippincott, 1959.

Oh, C. S.; Won, H. S.; Lee, K. S. \& Chung, I. H. Origin of the 
radial nerve branch innervating the brachialis muscle. Clin. Anat., 22:495-9, 2009.

Park, B. K.; Shin, Y. B.; Ko, H. Y.; Park, J. H. \& Baek, S. Y.Anatomic motor point localization of the biceps brachii and brachialis muscles. J. Korean Med. Sci., 22:459-62, 2007.

Pacha Vicente, D.; Forcada Calvet, P.; Carrera Burgaya, A. \& Llusá Pérez, M. Innervation of biceps brachii and brachialis: anatomical and surgical approach. Clin. Anat., 18:186-94, 2005 .

Poirier, P.; Charpy, A. \& Nicolas, A. Traité d'anatomie humaine. $4^{\circ}$ ed. Paris, Masson, 1901.

Prakash; Kumari, J.; Singh, N.; Rahul Deep, G.; Akhtar, T. \& Sridevi, N. S. A cadaveric study in the Indian population of the brachialis muscle innervation by the radial nerve. Rom. J. Morphol. Embryol., 50(1):111-4, 2009.

Rouvière, H. \& Delmas, A. Anatomía Humana: Descriptiva, Topográfica y Funcional. 11a ed. Madrid, Elsevier, 2005.

Spinner, R. J.; Pichelman, M. A. \& Birch, R. Radial nerve innervation to the inferolateral segment of the brachialis muscle: From anatomy to clinical reality. Clin. Anat., 16:3689, 2003.

Sunderland, S. The intraneural topograph of the radial, median and ulnar nerves. Brain, 68:243-98, 1945.

Testut, L. Les anomalies musculaires chez l' homme. Paris, G. Masson Editeur, 1884.

Testut, L. \& Latarjet, A. Tratado de anatomía humana. $8^{\text {va }}$ ed. Salvat, 1943. V. 1.

Williams, P. \& Warwick, R. Functional neuroanatomy of man. Philadelphia, W.B. Saunders Company, 1975.

Yan, J.; Aizawa, Y.; Honma, S. \& Horiguchi, M. Re-evaluation of the human brachialis muscle by fiber analysis of supply nerves. Kaibogaku Zasshi, 73:247-58, 1998.

\author{
Dirección para correspondencia: \\ Claudio Rodrigo Molina Osorio \\ Programa de Anatomía y Biología del Desarrollo \\ Facultad de Medicina \\ Universidad de Chile. \\ Santiago \\ CHILE \\ E-mail: claurod.mol@gmail.com
}

Recibido : 09-08-2011

Aceptado: 11-09-2011 